\title{
A systematic review of telehealth interventions for managing anxiety and depression in African American adults
}

\author{
Terika McCall ${ }^{1,2} \wedge$, Clinton S. Bolton III $^{3}$, Rebecca Carlson ${ }^{4} \wedge$, Saif Khairat ${ }^{1,5} \wedge$ \\ ${ }^{1}$ Carolina Health Informatics Program, University of North Carolina at Chapel Hill, Chapel Hill, NC, USA; ${ }^{2}$ Center for Medical Informatics, Yale \\ School of Medicine, New Haven, CT, USA; ${ }^{3}$ School of Education, University of North Carolina at Chapel Hill, Chapel Hill, NC, USA; ${ }^{4} \mathrm{Health}$ \\ Sciences Library, University of North Carolina at Chapel Hill, Chapel Hill, NC, USA; ${ }^{5}$ School of Nursing, University of North Carolina at Chapel \\ Hill, Chapel Hill, NC, USA \\ Contributions: (I) Conception and design: T McCall, S Kairat; (II) Administrative support: R Carlson; (III) Provision of study material or patients: \\ T McCall, R Carlson, S Kairat; (IV) Collection and assembly of data: T McCall; (V) Data analysis and interpretation: T McCall, CS Bolton 3rd, S \\ Kairat; (VI) Manuscript writing: All authors; (VII) Final approval of manuscript: All authors. \\ Correspondence to: Terika McCall, PhD, MPH, MBA. Yale School of Medicine, Center for Medical Informatics, 300 George Street, Suite 501, New \\ Haven, CT 06511, USA. Email: terika.mccall@yale.edu.
}

\begin{abstract}
Background: Although the difference in prevalence of mental illness is less than 7\%, African American adults utilize mental health services at less than half the rate of their white counterparts. Evidence from past studies showed that telehealth interventions for anxiety and depression are effective in reducing symptoms. The objective of this systematic review is to survey the available peer-reviewed literature for studies that used telehealth interventions, specifically tailored for African American adults, to reduce anxiety or depression, and determine their effectiveness.

Methods: A comprehensive literature search was conducted using the PubMed, PsycINFO, Scopus, and Web of Science electronic databases for relevant articles published from January 1970 to December 2019.

Results: Three independent studies were identified. The findings showed significant reduction of depressive symptoms post-intervention (all $\mathrm{P}<0.05$ ). However, effectiveness of telehealth intervention compared to face-to-face was not determined. None of the studies assessed the effectiveness of telehealth interventions to reduce anxiety.

Conclusions: The results highlight the need for additional research into the effectiveness of using telehealth modalities to manage anxiety and depression in African American adults. This systematic review has been registered in the PROSPERO international prospective register of systematic reviews (registration number: CRD42018104469; registration date: 09 August 2018).
\end{abstract}

Keywords: African Americans; telemedicine; anxiety; depression; systematic review

Received: 10 April 2020; Accepted: 18 September 2020; Published: 20 April 2021.

doi: $10.21037 /$ mhealth-20-114

View this article at: http://dx.doi.org/10.21037/mhealth-20-114

\section{Introduction}

Approximately $18 \%$ of African American adults (18 years and older) experienced mental illness in the last year (1). Although there is less than a $7 \%$ difference in the prevalence of mental illness among African Americans compared to white adults (24.6\%), African Americans utilize mental health services at less than half the rate of their white counterparts (8.9\% compared to $20.6 \%$ ) (1). Anxiety and depressive disorders are among the most common mental illnesses (2). Approximately $9 \%$ of non-Hispanic

^ ORCID: Terika McCall, 0000-0002-8143-5393; Rebecca Carlson, 0000-0002-4380-8435; Saif Khairat, 0000-0002-8992-2946. 
Black adults reported receiving a diagnosis of anxiety in their lifetime (3). Furthermore, 12.3\% of non-Hispanic Black adults reported receiving a diagnosis of depression in their lifetime (3). Historically, mental illness has been underreported in the African American community due to underdiagnosis and misdiagnosis; therefore, the true burden may actually be significantly higher than reported prevalence estimates.

Over $70 \%$ of African American adults that reported experiencing mental illness in the last year did not receive any mental health treatment during that time (1). There are many reasons why African Americans may not seek mental health services when needed. Barriers such as stigmatization of mental illness, less access to treatment, no or inadequate health insurance, mistrust of providers, and low health literacy prevent traditionally marginalized populations from seeking care $(4,5)$. Findings from previous studies showed that telehealth interventions for anxiety (6-13) and depression (7,9-20) are effective.

Use of telehealth modalities (e.g., video calls) has the potential to reduce access issues, such as, geographic proximity to a preferred mental health care professional (e.g., therapist). A recent study by McCall et al. found that over $70 \%$ of African American women endorsed the use of video calls to communicate with a professional to receive help to manage anxiety and depression (21). The convenience and familiarity of using telehealth modalities, coupled with use of proven psychotherapy treatments, such as cognitive behavioral therapy (CBT), make use of telehealth interventions feasible alternatives to traditional in-person treatment.

Successful interventions have used modalities such as telephone $(9,10,16)$, videoconferencing $(11,12,17)$, text messaging $(18,20)$, web-based formats (e.g., websites, email) $(6,15,22)$, and mobile applications $(13,19,23)$ to help participants reduce anxiety or depressive symptoms. However, the majority of the published studies were conducted with predominantly white study populations. Therefore, the results may not be generalizable to all racial groups, which suggests more investigation into the effectiveness of telehealth use with the African American adult population is needed. Furthermore, interventions should be culturally-tailored to meet the needs of the population to increase adoption, retention, and effectiveness. The objective of this systematic review is to survey the available peer-reviewed literature for studies that used telehealth interventions, specifically tailored for African American adults, to reduce anxiety or depression, and determine their effectiveness. We present the following article in accordance with the PRISMA reporting checklist (available at http://dx.doi. org/10.21037/mhealth-20-114).

\section{Methods}

\section{Search strategy}

Consistent with methods outlined in the PRISMA Statement for systematic reviews the search strategy consisted of three steps (24). First, a comprehensive literature search was conducted using the PubMed, PsycINFO, Scopus, and Web of Science electronic databases for relevant articles published from January 1970 to December 2019. A combination of keywords relating to African Americans, depression, anxiety, and telehealth were used. Table 1 provides an example of search terms and queries executed in PubMed. Second, reference lists of the included primary articles and retrieved systematic reviews were examined to identify any relevant publications. Finally, key technology journals (Fournal of Telemedicine and Telecare and Telemedicine and e-Health) were searched. The PRISMA flow diagram (24) for the review is shown in Figure 1. This systematic review has been registered in the PROSPERO international prospective register of systematic reviews (registration number: CRD42018104469; registration date: 09 August 2018). ${ }^{1}$

\section{Inclusion and exclusion criteria}

The criteria used for inclusion in this review were: (I) intervention targeted Black/African American adults ( $\geq 18$ years old); (II) primary outcome(s) include either diagnosis or symptom severity of depressive or anxiety disorder; (III) telehealth-based psychological intervention; (IV) intervention effectiveness evaluated using one or more standardized measures of anxiety or depression (e.g., Generalized Anxiety Disorder 7-item scale, Patient Health Questionnaire-9) administered pre- and post-intervention; and $(\mathrm{V})$ feasibility of using telehealth modality to receive psychological help (e.g., retention rate), acceptability of using telehealth modality to receive mental health services (e.g., satisfaction questionnaire), or self-management

\footnotetext{
${ }^{1}$ Available from: https://www.crd.york.ac.uk/prospero/display_record.php?ID=CRD42018104469.
} 
Table 1 Keywords and Boolean operators used in the PubMed database search

african americans[MeSH Terms] OR (african[ALL] AND americans[ALL]) OR african americans[ALL] OR blacks[ALL] OR african continental ancestry group[MeSH Terms] OR (african[ALL] AND continental[ALL] AND ancestry[ALL] AND group[ALL]) OR african continental ancestry group[ALL]

depression[ALL] OR depression[MeSH Terms] OR (Depressive[ALL] AND Symptom[ALL]) OR (Depressive[ALL] AND (syndrome[MeSH Terms] OR syndrome[ALL])) OR depressive disorder[MeSH Terms] OR (depressive[ALL] AND disorder[ALL]) OR "depressive disorder"[ALL]

anxiety[MeSH Terms] OR anxiety[ALL] OR (Social[ALL] AND (anxiety[MeSH Terms] OR anxiety[ALL])) OR hypervigilance[ALL] OR nervousness[ALL] OR ((anxiety[MeSH Terms] OR anxiety[ALL]) AND (disorder[ALL]))

((Mobile[ALL] AND (technology[MeSH Terms] OR technology[ALL])) OR (Mobile[ALL] AND (health[MeSH Terms] OR health[ALL])) OR m-health[ALL] OR telecounseling[ALL] OR (telemedicine[MeSH Terms] OR telemedicine[ALL]) OR (telemedicine[MeSH Terms] OR telemedicine[ALL] OR telehealth[ALL]) OR telepsych[ALL] OR telepsychiatry[ALL] OR (telemedicine[MeSH Terms] OR telemedicine[ALL] OR ehealth[ALL]) OR (Digital[ALL] AND (health[MeSH Terms] OR health[ALL])) OR (Text[ALL] AND Messaging[ALL]) OR (Text[ALL] AND Message[ALL]) OR (Mobile[ALL] AND Phone[ALL]) OR (Mobile[ALL] AND Phones[ALL]) OR (Mobile[ALL] AND Application[ALL]) OR (Mobile[ALL] AND Applications[ALL]) OR (Short[All Fields] AND Message[All Fields] AND Service[All Fields]) OR sms[ALL] OR ((multimedia[MeSH Terms] OR multimedia[ALL]) AND Messaging[ALL] AND Service[ALL]) OR mms[ALL] OR (Remote[ALL] AND(patients[MeSH Terms] OR patients[ALL] OR patient[ALL]) AND Monitoring[ALL]))

through participation in the intervention (e.g., patient follow-up survey) assessed post-intervention. Studies excluded from the review were (I) not peer-reviewed; (II) not an independent study (e.g., systematic review or metaanalysis); (III) not conducted in the USA; or (IV) used a pediatric population.

\section{Study evaluation}

\section{Study selection}

Covidence software was used for article screening and data extraction (25). Two independent reviewers (T.M. and C.B.) independently analyzed each title and abstract of articles retrieved to determine their relevance. The full text of potentially eligible studies was retrieved and similarly analyzed by T.M. and C.B. to exclude papers that did not meet inclusion criteria. Any disagreement over the eligibility of particular studies was resolved by an adjudicator (S.K.).

\section{Data extraction}

The data extraction template in the Covidence software was used (25). Extracted data included information on study setting, study population and participant demographics and baseline characteristics, details of the intervention, study methodology, recruitment and study completion rates, outcomes and times of measurement, indicators of acceptability to users, and suggested mechanisms of intervention action. Information for assessment of the risk of bias was included.

\section{Quality assessment}

The Critical Appraisal Skills Programme (CASP) checklists were used to assess the quality of the identified studies $(26,27)$. The checklists were developed and piloted by a group of experts for use in assessing the quality of various study types, therefore, they were deemed appropriate for use in this review. T.M. and C.B. independently assessed the risk of bias of all included studies. Disagreements between the reviewers over the risk of bias in particular studies were resolved by discussion, with involvement of an adjudicator (S.K.) where necessary. Intervention effectiveness was determined by significant reduction in depressive or anxiety symptoms post-intervention $(\mathrm{P}<0.05)$.

\section{Results}

\section{Overview of search results}

A comprehensive search in the selected citation databases identified a total of 689 articles. Twenty-one additional articles were identified through other sources (see search strategy section). After the removal of duplicates, 688 unique articles were included in the title and abstract screening process. The majority of the articles that were excluded did not involve psychological interventions that targeted African American adults. Sixty-four papers were identified as relevant to the research question and included in the full-text screening process. During the independent full-text screening process, 21 articles were identified as having the wrong study design, 19 were not conducted in the U.S., 16 had the wrong patient population, and five 


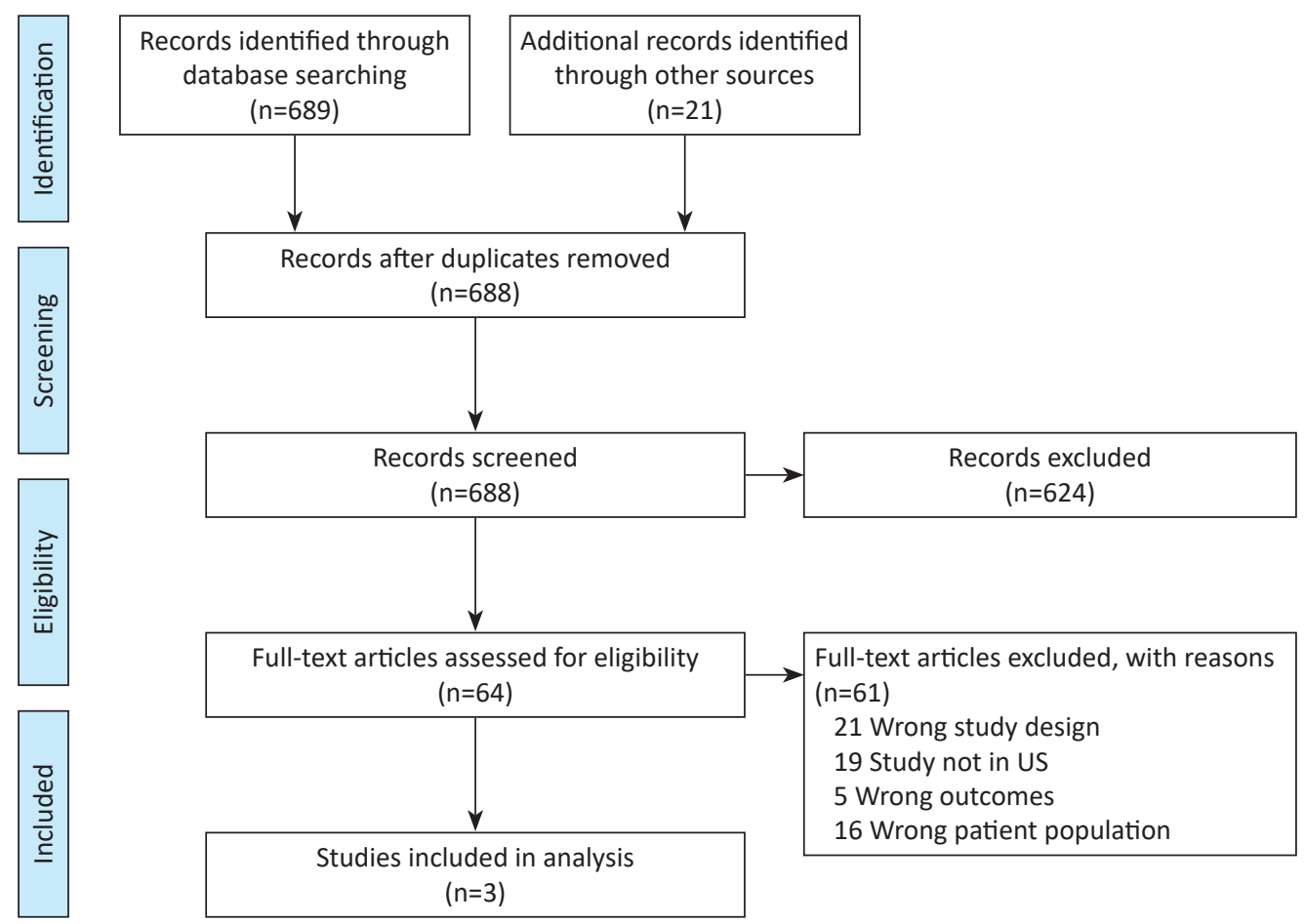

Figure 1 PRISMA flow diagram of search strategy.

focused on the wrong primary outcomes. Three articles were identified that met the inclusion criteria and were included in the analysis (28-30). The PRISMA diagram (24) for the review is shown in Figure 1. Summaries of study characteristics are provided in Table 2.

The three independent studies had a pooled total of 32 participants (range 6-15 per study). Only one of the studies was a randomized controlled trial (RCT) (28). The remaining two studies were prospective cohort studies assessing the feasibility of interventions $(29,30)$. Two of the studies reported a majority $(>80 \%)$ of their sample were female $(28,30)$. One study had all HIVpositive participants (30), and another reported $60 \%$ of participants were HIV-positive (29). All of the studies used validated assessments [i.e., the Center for Epidemiological Studies Depression Scale (CES-D) $(28,29)$ or Hamilton Depression Rating Scale (HAM-D) (30)] to assess the primary outcome of depression pre and post intervention.

The randomized controlled trial by Glueckauf et al. focused on African American caregivers (CG) with depression (28). Telephone-based CBT was used for the treatment group and traditional face-to-face CBT for controls. The content and structure of the CBT intervention was the same for both groups; 12 weekly one- hour sessions led by a trained counselor (7 group sessions and 5 individual goal setting and implementation sessions). The telephone-based CBT sessions occurred in the participant's home via a teleconferencing system, and the face-to-face sessions were performed in a conference room at a university or in a private room in a public library.

Similarly, the prospective cohort study by Himelhoch et al. also utilized telephone-based CBT for an intervention targeting urban dwelling HIV-positive African Americans with major depression (30). The structure of the intervention comprised of 11 sessions, delivered over 14 weeks by a trained therapist (one initial evaluation session, five behavioral activation sessions, and five cognitive restructuring sessions). All sessions were scheduled in advance, and participants were reminded to select a private and confidential place to participate in the CBT session. The calls lasted from 27-70 minutes.

In contrast, the prospective cohort study by HightowWeidman et al. used a peer support intervention for both young Black men-who-have-sex-with-men (MSM) and transgender women (29). Participants were asked to spend at least one hour per week on the mobile phone-optimized site (HealthMpowerment.org) for four weeks. The aims of the site were to promote health and wellness and serve as a 


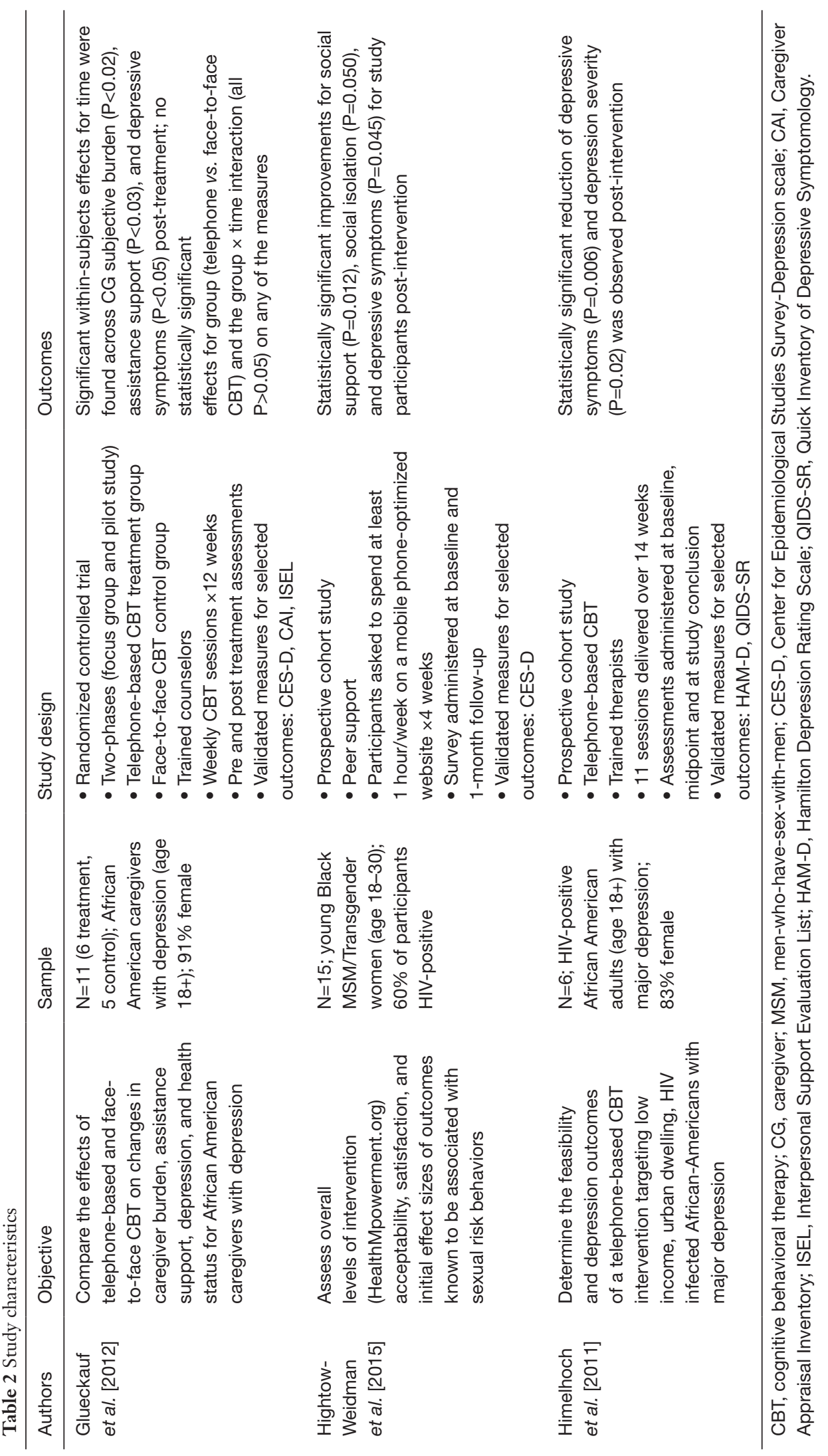


platform for social support.

\section{Quality assessment}

All three studies (28-30) met a majority of the criteria used for consideration in the assessment of study quality in the CASP appraisal checklists (26,27) (i.e., reviewers T.M. and C.B. answered 'yes' for $>80 \%$ of the questions). Two studies lacked control groups $(29,30)$, and all had small sample sizes (6-15 participants) and short follow-up periods ( $<6$ months) (28-30). This resulted in the reviewers rating all of the studies to be of 'fair' quality. However, all studies were published in peer reviewed journals and deemed appropriate for inclusion.

\section{Outcomes}

Statistically significant reduction of depressive symptoms (measured by improvement in mean CES-D or HAM-D score) was observed post-intervention in all of the studies $(\mathrm{P}<0.05)(28-30)$. In addition, one study showed a significant reduction in depression severity post-intervention $(\mathrm{P}=0.02$, measured by improvement in mean Quick Inventory of Depressive Symptomology (QIDS-SR) score) (30). All studies reported patient satisfaction with the interventions (28-30). Hightow-Weidman et al. also reported improvements for social support $(\mathrm{P}=0.012)$ and social isolation $(\mathrm{P}=0.050)$ for study participants post-intervention (29).

Furthermore, the RCT conducted by Glueckauf et al. found significant within-subjects effects for time across CG subjective burden $[\mathrm{P}<0.02$, measured by improvement in the subjective burden subscale of the Caregiver Appraisal Inventory $(\mathrm{CAI})]$ and assistance support $[\mathrm{P}<0.03$, measured by improvement in the Assistance subscale of Interpersonal Support Evaluation List (ISEL)] post-treatment (28). However, no statistically significant effects were observed for group (telephone vs. face-to-face CBT) and the group $\mathrm{x}$ time interaction (all $\mathrm{P}>0.05$ ) on any of the measures (28). The effect sizes for changes in physical symptoms over the past month for both treatment groups were small, however improvement in physical symptoms post-treatment was somewhat higher for CGs in the telephone CBT group than those in the face-to-face CBT group (28).

\section{Discussion}

\section{Principal findings}

To our knowledge, this is one of the first systematic reviews to examine studies aimed at managing anxiety or depression in African American adults through use of culturally-tailored telehealth interventions. None of the three studies included in the review assessed the effectiveness of telehealth interventions to reduce anxiety. The results of the review showed significant reduction of depressive symptoms (28-30) and depression severity post-intervention (30) (all $\mathrm{P}<0.05$ ). One study also found a significant increase in social support post-intervention ( $\mathrm{P}=0.012)$ (29). However, in the RCT, the effectiveness of a telehealth intervention compared to faceto-face was not determined (28). Telehealth intervention satisfaction and retention was high among participants $(>75 \%)(28-30)$.

These findings are consistent with literature on the use of telehealth interventions to manage depression in other populations. Previous studies have also found the use of telehealth interventions to be effective in reducing depressive symptoms (all $\mathrm{P}<0.05)$ among predominantly white (9-11,13-15,19,22,23), Latino (16,31-33), Hispanic $(31,32)$, and Chinese (33) participants. Overall, participants reported satisfaction with the telehealth interventions.

The scarcity of peer-reviewed published research on the use of telehealth modalities for management of anxiety and depression in African American adults is highlighted in this review. Research in this area is premature for African American adults in comparison to other populations, which indicates the need for additional research on the use of telehealth modalities for anxiety and depression management with this group. Only three studies were identified, and all of them were pilot trials assessing the feasibility of the telehealth interventions (28-30).

\section{Strengths and limitations}

The main strengths of this review are the systematic search strategy used, and independent screening and quality assessment of the articles by two reviewers (with involvement of an adjudicator where necessary). However, there were limitations which should be considered when interpreting the findings. The main limitations were the number of articles identified $(n=3)$, small sample sizes (range 6-15 participants per study), and potential publication bias due to the exclusion of grey literature. Two of the three studies lacked control groups $(29,30)$, which limits the ability to make inferences regarding the efficacy of the interventions. Due to the difference in study types (RCT vs. prospective cohort study), a quantitative analysis and comparison of treatment effectiveness across 
studies was not feasible. Also, the generalizability of the findings is limited due to the majority of participants in the studies having a specific health condition (e.g., HIVpositive) $(29,30)$ or special circumstance (e.g., caregivers of dementia patients) (28).

\section{Conclusions and future directions}

Future research should investigate the effectiveness of using telehealth interventions to manage anxiety and depression in more generalizable samples of African American adults. Furthermore, studies should include larger sample sizes, control groups, longer intervention periods, and followup assessments to determine the long-term sustainability of using telehealth modalities to manage anxiety and depression. Socio-cultural factors that promote or discourage positive coping skills and help-seeking behavior should be considered by researchers and clinicians when designing interventions and providing treatment. The population should also be surveyed to determine acceptable modalities to receive care. For example, a recent survey of African American women revealed that video call was an acceptable modality to communicate with a professional to receive help for managing anxiety and depression (21), whereas text messaging was not (34).

Taking into account the burden of unmet need and disparity in mental health service utilization among African American adults, there is great potential to use telehealth modalities to deliver services to this population. Telehealth is proving to be acceptable for some mental health services, such as CBT $(9,28,30)$. Given that $75 \%$ of African American adults own a smartphone (35), the use of smartphones to deliver interventions to this population needs to be explored further. This presents an opportunity to create innovative interventions that increase access to much needed mental health services. However, to increase the likelihood of adoption among African American adults, telehealth interventions should be culturally-tailored. A "one-size-fitsall" approach to designing telehealth interventions to help African American adults manage anxiety or depression may lead to more options but continued disparity in receiving care. While the use of telehealth is gaining traction, more research is needed on the acceptability and effectiveness of using various modalities (e.g., video call, mobile apps) with traditionally underserved populations so that harm is minimized and all benefit from advances in mental health care. Only then can we achieve digital health equity by providing tools that addresses the specific needs and interests of African American adults.

\section{Acknowledgments}

We are grateful to Drs. Paul Fontelo, Javed Mostafa, Enrique Neblett, Jr., and Fei Yu for reviewing this systematic review.

Funding: The first author was supported by funding from the National Library of Medicine's Institutional Training Grant for Research Training in Biomedical Informatics and Data Science at the Carolina Health Informatics Program (T15-LM012500) and Yale Center for Medical Informatics (T15-LM007056).

\section{Footnote}

Reporting Checklist: The authors have completed the PRISMA reporting checklist. Available at http://dx.doi. org/10.21037/mhealth-20-114

Conflicts of Interest: All authors have completed the ICMJE uniform disclosure form (available at http://dx.doi. org/10.21037/mhealth-20-114). The authors have no conflicts of interest to declare.

Ethical Statement: The authors are accountable for all aspects of the work in ensuring that questions related to the accuracy or integrity of any part of the work are appropriately investigated and resolved.

Open Access Statement: This is an Open Access article distributed in accordance with the Creative Commons Attribution-NonCommercial-NoDerivs 4.0 International License (CC BY-NC-ND 4.0), which permits the noncommercial replication and distribution of the article with the strict proviso that no changes or edits are made and the original work is properly cited (including links to both the formal publication through the relevant DOI and the license). See: https://creativecommons.org/licenses/by-nc-nd/4.0/.

\section{References}

1. Substance Abuse and Mental Health Services Administration. (2019). National Survey on Drug Use and Health, 2018 [data file]. Research Triangle Park, NC: RTI International [distributor]. Accessed 21 February 2020. Available online: https://www.samhsa.gov/data.

2. Kessler RC, Berglund P, Demler O, et al. Lifetime 
prevalence and age-of-onset distributions of DSM-IV disorders in the National Comorbidity Survey Replication. Arch Gen Psychiatry 2005;62:593-602.

3. Reeves WC, Strine TW, Pratt LA, et al. Mental Illness Surveillance Among Adults in the United States. MMWR Suppl 2011;60:1-29.

4. Thompson VLS, Bazile A, Akbar M. African americans' perceptions of psychotherapy and psychotherapists. Professional Psychology: Research and Practice 2004;35:19-26.

5. National Alliance on Mental Illness. Multicultural Mental Health Facts. Retrieved 13 November 2018. Available online: https://www.nami.org/Learn-More/MentalHealth-By-the-Numbers

6. Musiat P, Conrod P, Treasure J, et al. Targeted prevention of common mental health disorders in university students: randomised controlled trial of a transdiagnostic traitfocused web-based intervention. PLoS One 2014;9:e93621.

7. Cukrowicz KC, Joiner TE Jr. Computer-Based Intervention for Anxious and Depressive Symptoms in a Non-Clinical Population. Cognit Ther Res 2007;31:677-93.

8. Ivanova E, Lindner P, Ly KH, et al. Guided and unguided Acceptance and Commitment Therapy for social anxiety disorder and/or panic disorder provided via the Internet and a smartphone application: A randomized controlled trial. J Anxiety Disord 2016;44:27-35.

9. Brenes GA, Danhauer SC, Lyles MF, et al. TelephoneDelivered Cognitive Behavioral Therapy and TelephoneDelivered Nondirective Supportive Therapy for Rural Older Adults With Generalized Anxiety Disorder: A Randomized Clinical Trial. JAMA Psychiatry 2015;72:1012-20.

10. Mavandadi S, Benson A, DiFilippo S, et al. A TelephoneBased Program to Provide Symptom Monitoring Alone vs Symptom Monitoring Plus Care Management for LateLife Depression and Anxiety: A Randomized Clinical Trial. JAMA Psychiatry 2015;72:1211-8.

11. Dunstan DA, Tooth SM. Treatment via videoconferencing: a pilot study of delivery by clinical psychology trainees. Aust J Rural Health 2012;20:88-94.

12. Yuen EK, Herbert JD, Forman EM, et al. Acceptance based behavior therapy for social anxiety disorder through videoconferencing. J Anxiety Disord 2013;27:389-97.

13. Proudfoot J, Clarke J, Birch MR, et al. Impact of a mobile phone and web program on symptom and functional outcomes for people with mild-to-moderate depression, anxiety and stress: a randomised controlled trial. BMC
Psychiatry 2013;13:312.

14. Buntrock C, Ebert DD, Lehr D, et al. Effect of a WebBased Guided Self-help Intervention for Prevention of Major Depression in Adults With Subthreshold Depression: A Randomized Clinical Trial. JAMA 2016;315:1854-63.

15. Morgan AJ, Jorm AF, Mackinnon AJ. Email-based promotion of self-help for subthreshold depression: Mood Memos randomised controlled trial. Br J Psychiatry 2012;200:412-8.

16. Dwight-Johnson M, Aisenberg E, Golinelli D, et al. Telephone-based cognitive-behavioral therapy for Latino patients living in rural areas: a randomized pilot study. Psychiatr Serv 2011;62:936-42.

17. Choi NG, Hegel MT, Marti N, et al. Telehealth problemsolving therapy for depressed low-income homebound older adults. Am J Geriatr Psychiatry 2014;22:263-71.

18. Agyapong VIO, Juhás M, Ohinmaa A, et al. Randomized controlled pilot trial of supportive text messages for patients with depression. BMC Psychiatry 2017;17:286.

19. Arean PA, Hallgren KA, Jordan JT, et al. The use and effectiveness of mobile apps for depression: results from a fully remote clinical trial. J Med Internet Res 2016;18:e330.

20. Aguilera A, Berridge C. Qualitative feedback from a text messaging intervention for depression: benefits, drawbacks, and cultural differences. JMIR Mhealth Uhealth 2014;2:e46.

21. McCall T, Schwartz T, Khairat S. Acceptability of telemedicine to help African American women manage anxiety and depression. Stud Health Technol Inform 2019;264:699-703.

22. Clarke G, Reid E, Eubanks D, et al. Overcoming depression on the Internet (ODIN): a randomized controlled trial of an Internet depression skills intervention program. J Med Internet Res 2002;4:E14.

23. Clarke J, Proudfoot J, Birch MR, et al. Effects of mental health self-efficacy on outcomes of a mobile phone and web intervention for mild-to-moderate depression, anxiety and stress: secondary analysis of a randomised controlled trial. BMC Psychiatry 2014;14:272.

24. Moher D, Liberati A, Tetzlaff J, et al. Preferred reporting items for systematic reviews and meta-analyses: the PRISMA statement. PLoS Med 2009;6:e1000097.

25. Veritas Health Innovation. Covidence systematic review software. Melbourne, Australia. Available online: www. covidence.org

26. Critical Appraisal Skills Programme. CASP Randomised 
Controlled Trial Checklist. Available at https://casp-uk. net/casp-tools-checklists/. Accessed: 23 August 2018.

27. Critical Appraisal Skills Programme. CASP Cohort Study Checklist. Available online: https://casp-uk.net/casp-toolschecklists/. Accessed: 23 August 2018.

28. Glueckauf RL, Davis WS, Willis F, et al. Telephonebased, cognitive-behavioral therapy for African American dementia caregivers with depression: initial findings. Rehabil Psychol 2012;57:124-39.

29. Hightow-Weidman LB, Muessig KE, Pike EC, et al. HealthMpowerment.org: Building Community Through a Mobile-Optimized, Online Health Promotion Intervention. Health Educ Behav 2015;42:493-9.

30. Himelhoch S, Mohr D, Maxfield J, et al. Feasibility of telephone-based cognitive behavioral therapy targeting major depression among urban dwelling African-American people with co-occurring HIV. Psychol Health Med 2011;16:156-65.

doi: 10.21037/mhealth-20-114

Cite this article as: McCall T, Bolton CS 3rd, Carlson R, Khairat S. A systematic review of telehealth interventions for managing anxiety and depression in African American adults. mHealth 2021;7:31.
31. Chong J, Moreno F. Feasibility and acceptability of clinicbased telepsychiatry for low-income Hispanic primary care patients. Telemed J E Health 2012;18:297-304.

32. Moreno FA, Chong J, Dumbauld J, et al. Use of standard Webcam and Internet equipment for telepsychiatry treatment of depression among underserved Hispanics. Psychiatr Serv 2012;63:1213-7.

33. Choi I, Zou J, Titov N, et al. Culturally attuned Internet treatment for depression amongst Chinese Australians: a randomised controlled trial. J Affect Disord 2012;136:459-68.

34. McCall T, Schwartz TA, Khairat S. The Acceptability of Text Messaging to Help African American Women Manage Anxiety and Depression: Cross-Sectional Survey Study. JMIR Ment Health 2020;7:e15801.

35. Pew Research Center. Mobile Fact Sheet. Retrieved 15 November 2018. Available online: http://www. pewinternet.org/fact-sheet/mobile/ 